\title{
Hyper-Branched Dendrimers in Drug Delivery and Solubilization
}

\author{
Umesh Gupta* \\ Department of Pharmacy, School of Chemical Sciences and Pharmacy, Central University of Rajasthan, India
}

Received: March 11, 2014, Accepted: August 22, 2014, Published: August 27, 2014

*Corresponding author: Umesh Gupta, Department of Pharmacy, School of Chemical Sciences and Pharmacy, Central University of Rajasthan, NH-8 Jaipur Ajmer Expressway, BandarsindriAjmer Rajasthan - 305801India, Tel: +91-8003274082; E-mail: umeshgupta175@gmail.com; umeshgupta@ curaj.ac.in

\section{Keywords: PAMAM; Dendrimers; Solubilization}

Recent decades have witnessed the fascinating era of nanotechnology and nanomedicine in drug delivery and targeting. So many nanotechnology based formulations are either in the market today or else they are in the final stage of commercialization. Among different nano-carriers dendrimers represents an exceptionally versatile class of bio-macromolecules which has shown immense potential due to its hyper-branched structure and tailor-made functionality. Dendrimers are unimolecular, three dimensional, hyper branched monodispersed macromolecules, which can be obtained by an iterative sequence of reaction steps producing a unique and precise, branching structure which mimics tree like pattern. Reported in 1985 by Tomalia and coworkers, dendrimers consist of three characteristic scaffolds i.e. multi-functional initiator core, inner generations (or branches which consist of repeating branched units); and exterior or terminal surface groups (functional groups attached to the outermost generation)[1]. Polyamidoamine (PAMAM), polylysine, triazine and polypropyleneimine (PPI) represents the important classes of dendrimers. Dendrimers can be synthesized using either convergent or divergent strategies. Unlike the traditional polymers which often have poorly defined structure, the divergent and convergent approaches of dendrimer synthesis can be precisely controlled at each step. The basic difference between convergent and divergent strategies lies in the growth and propagation of dendrimers during synthesis [2].

In recent years dendrimers have proved its versatility in different biomedical areas such as drug delivery, gene delivery, diagnosis and solubilization. The properties of dendrimers which contributes in its potential of drug delivery and solubilization includes excellent host guest chemistry, monodispersity, unimicellar nature and tunable surface functional groups. Usually a generation dependent change in properties can be observed. In general the lower generation dendrimers (1-3) have open structure with highly asymmetric shape compared to higher generations in which the structure is more and more globular and compact (higher then generation 4). At higher generation, dendrimers become densely packed as they extend out to the periphery, which forms a closed membrane-like structure [3]. When a critical stage is reached dendrimers cannot grow because of steric hindrance. This is sometimes referred to as 'starburst effect'. In case of PAMAM dendrimers the starburst effect can be observed after the tenth generation [4]. Number of terminal functional groups also increases exponentially with the increased branch density. These internal cavities and terminal functional groups play important role in controlling the properties of therapeutic moieties that are encapsulated or complexed with it [5].

The internal architecture of dendrimers is usually hydrophobic while the outer surface is hydrophilic in nature. This contributes to their solubilization property. More than 30 different hydrophobic drugs have been reported to be solubilized successfully using different dendrimers e.g. Indomethacin, Nifedipine, Paclitaxel, Famotidine, Amphotericin B, Niclosamide etc [6]. The hydrophilic exterior and hydrophobic interior is also responsible for their behavior as stable unimolecular micelle. The surface functionality contributes to their targeting and tunable properties. The surface of dendrimers can be engineered or modified using number of moieties such as ligand, antibodies, drugs etc. The solubilization is the result of the electrostatic interactions, hydrogen bonding and van der waals attraction between hydrophobe and surface functional groups of dendrimer [7].

\section{References}

1. Tomalia DA, Baker H, Dewald JR, Hall M, Kallos G, S Martin, et al. A new class of polymers: Starburst- dendritic macromolecules. Polymer Journal. 1985; 17:117-132. doi:10.1295/polymj.17.117.

2. Gupta U. Dendrimers and its Biomedical Applications. In: Sangamesh Kumbar, Cato Laurencin, Meng Deng, editors. Natural and Synthetic Biomedical Polymers. 1st ed. San Diego: Elsevier Inc; 2014. pp. 243257.

3. Caminade AM, Laurent R, Majoral JP. Characterization of dendrimers. Adv Drug Deliv Rev. 2005; 57 (15): 2130-2146.

4. Fischer M, Vögtle F. Dendrimers: From design to applications - A progress report. Angewandte Chemie International Edition. 1999; 38(7): 884-905. 
5. Tomalia DA, Naylor AM, Goddard WA. Starburst Dendrimers: Molecular-Level Control of Size Shape Surface Chemistry Topology and Flexibility from Atoms to Macroscopic Matter. Angew Chem Int Ed Engl. 1990; 29(2):138- 175. doi: 10.1002/anie.199001381.

6. Gupta U, Agashe HB, Asthana A, Jain NK. Dendrimers: novel polymeric nano-architectures for solubility enhancement. Biomacromolecules. 2006; 7(3):649-658.

7. Satija J, Gupta U, Jain NK. Pharmaceutical and biomedical potential of surface engineered dendrimers. Crit Rev Ther Drug Carr Syst. 2007; 24(3):257-306. 